\title{
IMPACTOS SOCIOAMBIENTALES DEL CULTIVO DE PALMA AFRICANA: LOS CASOS MEXICANO Y BRASILEÑO
}

\author{
SOCIO-ENVIRONMENTAL IMPACTS OF AFRICAN OIL PALM CULTIVATION: \\ MEXICAN AND BRAZILIAN CASES
}
IMPACTOS SOCIOAMBIENTAIS DO CULTIVO DO DENDEZEIRO: OS CASOS MEXICANO E BRASILEIRO

\section{Agustín Ávila Romero ${ }^{1}$ Jadson Albuquerque ${ }^{2}$}

\begin{abstract}
Resumen
Con el presente artículo buscamos analizar los impactos socioambientales de la territorialización de la palma africana (Elaeis guineensis) en México y en Brasil. Dicho cultivo es fuertemente impulsado por empresas agroindustriales y políticas públicas que han construido los respectivos Estados nación. La expansión de las plantaciones de palma africana no es un fenómeno solamente mexicano o brasileño, pues se encuentra presente en diversas partes del mundo, destacando su producción en países asiáticos, africanos y latinoamericanos. En México y Brasil las plantaciones destinadas a la producción del agrocombustible y derivados de la industria aceitera están vinculadas a la supuesta generación de ingresos económicos para los productores. En el caso brasileño, se les ha vinculado a la agenda de "inclusión social y económica" de la agricultura familiar, que presupone algunas políticas públicas, como es el caso del Programa Nacional de Producción y Uso de Biodiesel (PNPB) y el Programa de Producción Sustentable del Aceite de Palma (PPSOP). En el caso mexicano, se ha impulsado a través del Programa de Desarrollo
\end{abstract}

\section{Doi: http://dx.doi.org/10.15359/eys.23-53.4}

Fecha de recepción: 06-03-2018. Fechas de reenvíos: 23-04-2018, 08-05-2018. Aceptado el 17-05-2018. Publicado el 01-06-2018.

${ }^{1}$ Universidad Intercultural de Chiapas (UNICH), San Cristóbal de las Casas, Chiapas, México. Profesor Visitante Instituto de Estudios Socioambientales UFG/Brasil, Profesor Titular UNICH. Correo electrónico: agustinavila72@gmail.com

${ }^{2}$ Estudiante de maestría en Geografía por la Universidad Federal de Goiás (UFG), graduado en Geografía por la Universidad del Estado de Pará (UEPA). Instituto de Estudios Socio-ambientales (IESA), Universidad Federal de Goiás (UFG), BRASIL. Correo electrónico: albuquerqueson@gmail.com

62

Agustín Ávila Romero y Jadson Albuquerque

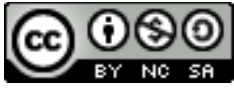

Revista Economía y Sociedad by Universidad Nacional is licensed under a CreativeCommons Reconocimiento-NoComercial- 
Productivo del Sur-sureste de FIRCO y FomenPalma. Los efectos muestran un modelo de acumulación basado en la desvalorización de la vida campesina, la explotación y el despojo agrario, provocando la reestructuración de los procesos de producción agropecuarios en México y en Brasil. La referencia temática hace parte de la agenda de investigación de los autores con una gran cantidad de lecturas sobre el tema y trabajos de campo en los dos países, entre los años 2014 y 2017.

Palabras claves: impactos socioambientales; palma africana; campesinado; México; Brasil

\begin{abstract}
Socio-environmental impacts of the territorialization of African oil palm (Elaeis guineensis) are analyzed in Mexico and Brazil. This crop is strongly promoted by agro-industrial companies and the public policies created by the respective Nation States. The expansion of African oil palm plantations is not solely a Mexican or Brazilian phenomenon, since it is present in different parts of the world, mainly being produced in Asia, Africa and Latin America. In Mexico and Brazil, agrofuel and oil derivative plantations are related to an assumed generation of economic income for producers. In Brazil, they have been linked to the "social and economic inclusion" agenda in family farming, which presupposes certain public policies, such as the National Program for the Production and Use of Biodiesel (PNPB) and the Sustainable Palm Oil Production Program (PPSOP). In Mexico, it has been promoted through FIRCO's South-Southeastern Productive Development Program and FomenPalma. Effects show an accumulation model based on the devaluation of the farmer's life, exploitation and agrarian dispossession, which causes the restructuring of agricultural production processes in Mexico and Brazil. References included an extensive number of texts on the subject and fieldwork in the two countries between 2014 and 2017.
\end{abstract}

Keywords: Socioenviromental impacts; African oil palm; Farmer; Mexico; Brazil.

\title{
Resumo
}

Com este artigo procuramos analisar os impactos socioambientais da territorialização do dendezeiro (Elaeis guineensis) no México e no Brasil. Esta safra é fortemente impulsionada por empresas agroindustriais e políticas públicas que foram construídas pelos respectivos estados-nação. A expansão das plantações de dendezeiros 
não é apenas um fenômeno mexicano ou brasileiro, pois está presente em diferentes partes do mundo, destacando sua produção em países asiáticos, africanos e latino-americanos. No México e no Brasil as plantações destinadas à produção do agrocombustível e derivados da indústria petrolífera estão ligadas à suposta geração de renda econômica para os produtores. No caso brasileiro, foi vinculado à agenda de "inclusão social e econômica" da agricultura familiar, o que pressupõe algumas políticas públicas, como é o caso do Programa Nacional de Produção e Uso do Biodiesel (PNPB) e do Programa de Produção Sustentável de Óleo de Palma (PPSOP). No caso mexicano, foi promovido através do Programa de Desenvolvimento Produtivo Sul/Sudeste da FIRCO e FomenPalma. Os efeitos mostram um modelo de acumulação baseado na desvalorização da vida campestre, exploração e desapropriação agrária, causando a reestruturação dos processos de produção agrícola no México e no Brasil. A referência temática faz parte da agenda de pesquisa dos autores com um grande número de leituras sobre o tema e trabalhos de campo nos dois países, entre 2014 e 2017.

Palavras-chave: impactos socioambientais; dendezeiro; campestre; México.

\section{Introducción}

La expansión del cultivo de la palma africana en las últimas décadas está relacionada básicamente con dos factores: con la amplia posibilidad de utilizar el aceite de palma o palm oil, como es conocido en el mercado internacional, sobre todo en la industria de alimentos y farmacéuticas; y con su utilización para generar energía por medio del agrocombustible. Según el Departamento de Agricultura de Estados Unidos (2017), la producción mundial de aceite de palma pasó de 33 millones de toneladas en 2004 a 66 millones de toneladas en 2016. Simultáneamente al crecimiento de la producción, aumentan también las denuncias referentes a los efectos socioambientales negativos provocados por el sector de la palma alrededor del mundo. A pesar de que el $21 \%$ de la producción mundial -equivalente a 2.51 millones de hectáreas y 11.97 millones de toneladas de aceite- está certificado por la Roundtable On Sustainable Palm Oil (RSPO, 2017), organización sin fines de lucro compuesta por varios productores alrededor del mundo, la palma africana es la principal causa de deforestación y violación de derechos de los campesinos e indígenas en Malasia e Indonesia ( países responsables de más del $80 \%$ de la producción mundial), de acuerdo con el Informe de Desarrollo Humano del Programa de las Naciones Unidas para el Desarrollo (2007). Y de su vinculación a grupos paramilitares que hacen uso de la violencia para el despojo de tierras en Honduras, Colombia y Guatemala (Rey, 2013). Así pues, en este contexto se describe la actual producción de palma africana en algunas partes del mundo. En México y Brasil, 
específicamente, hay una notable presencia del Estado y la presencia de campesinos en el proceso productivo.

En este trabajo nos proponemos analizar los arreglos del capital y de las instituciones acerca del cultivo de palma africana, así como sus impactos en diferentes dimensiones: social, ambiental, cultural, etc., tanto en México como Brasil. Para ello, realizamos nuestro análisis a partir de la idea de que el actual cultivo de palma, a escala mundial, se basa en sus prácticas productivas por depredar el medio ambiente como es el modelo agro-energético capitalista dominante que, a su vez, está directamente ligado a la mundialización de la agricultura capitalista, sometiendo los espacios rurales a los imperativos de grandes corporaciones del agronegocio.

Tanto en el caso brasileño como mexicano, la política pública integra la agricultura familiar campesina en la cadena productiva de la palma para la producción del agrocombustible a partir de esta oleaginosa.

Esta investigación permite realizar un análisis comparado del cultivo de palma africana en las dos principales economías de América Latina, líderes, a su vez, en la producción agrícola del continente. Además, ello es relevante ya que los grupos empresariales que se han constituido en ambas naciones actúan a escala regional en el continente e impactan dos de los principales ecosistemas de América Latina.

Brasil es el país más poblado de Latinoamérica, con 190 millones de habitantes, según el Instituto Brasileño de Geografía y Estadística (IBGE) en el Censo de 2010 (IBGE, 2010). Además, es la mayor economía del subcontinente y, según estudios de diversas instituciones, estará en el siglo XXI entre las principales cinco economías del mundo -actualmente es considerada la séptima economía del planeta-. México, por su parte, según el Instituto Nacional de Estadística y Geografía (INEGI), tiene una población estimada para el 2017 de 124 millones personas (INEGI, 2018), lo que lo convierte en el país hispanoparlante más poblado del planeta y la principal economía, se encuentra ya clasificada como la 13a economía del planeta.

Ahora bien, sus dinámicas agrarias son profundamente distantes. México tuvo un proceso de reforma agraria en el siglo XX que les permitió el acceso a la tierra a millones de campesinos e indígenas, mientras que Brasil, pese a las grandes luchas que han emprendido sus sujetos agrarios, es uno de los países con mayor desigualdad en el acceso a la tierra, que se encuentra concentrada en manos de pocos empresarios capitalistas.

Brasil ha tenido un crecimiento espectacular de su producción agrícola desde la década de los 80, colocándose como el tercer exportador mundial, solo detrás de Estados Unidos y la Unión Europea. Sus principales productos agrícolas de exportación son el café, la soya, la caña de azúcar y la carne bovina. México, por su parte, tiene una economía abierta en el sector agrícola, lo que ha significado una importación cada vez más grande de los alimentos básicos para el consumo de su población, como son los cereales (maíz, trigo y arroz) y el impulso a las 
plantaciones de frutales (plátano, papaya, limón, aguacate y mango) y hortalizas (el tomate, cebolla, brócoli y ajo), cuyo destino principal es Estados Unidos, al cual se dirigen cerca del $60 \%$ de las exportaciones.

Brasil ha estado enfrentándose en los últimos años a una caída de sus ingresos en el sector agrícola debido a la disminución de los precios; México, al contrario, ha aprovechado el Tratado de Libre Comercio (TLCAN) con EUA y Canadá y ha aumentado sus ingresos agrícolas con la exportación de productos hortícolas y frutales. Así pues, es bajo este contexto que ambas naciones ven en las plantaciones de palma africana la posibilidad de abastecer a los complejos agroindustriales que se construyen respectivamente, con base en programas gubernamentales. Tanto en Brasil como en México los palmicultores empresariales realizan diferentes actividades de gestión que les permiten incrementar notablemente su producción y recibir apoyos importantes en el proceso de transformación y comercialización.

Ubicar los efectos socioambientales que ocasionan dichas actividades es el principal motivo de nuestra pesquisa. Esta investigación permite visualizar dinámicas socioespaciales que impactan claramente los equilibrios ecológicos de las regiones donde se impulsan estas plantaciones, ocasionando problemas de contaminación del agua y el aire por el uso de agroquímicos, procesos de deforestación, subordinación o eliminación de la economía campesina y dominio de grupos empresariales en la producción de palma africana.

Tanto en México como en Brasil el crecimiento de esta producción es espectacular, llegando, en el caso mexicano, a cerca de las 100.000 hectáreas y, en el caso brasileño, por arriba de las 400.000 hectáreas. Ello es importante porque, además, este monocultivo se impulsa en las regiones tropicales mesoamericanas y en la Amazonia, que aportan la mayor cantidad de servicios ecosistémicos al continente. En ambos países ubicamos cómo las políticas públicas de gobiernos de diferentes espectros ideológicos han favorecido el impulso de la palma africana a través del subsidio o financiamiento de las actividades.

\section{Procedimientos metodológicos}

Los análisis realizados en este estudio provienen de tres procesos que se complementan: el levantamiento bibliográfico, las fuentes secundarias y el trabajo de campo.

El levantamiento bibliográfico tuvo por objetivo alimentar una base teórica por medio de la utilización de conceptos que permiten el análisis de la territorialización de la palma africana y de sus efectos como tema de investigación científica.

Los datos secundarios, por su parte, contribuyeron con la información de la cantidad producida de aceite de palma en los dos países investigados; además, posibilitaron una visión acerca de la estructura agraria y de la producción de palma africana en los territorios mexicano y brasileño.

66

Agustín Ávila Romero y Jadson Albuquerque

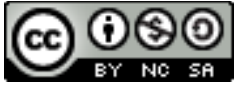

Revista Economía y Sociedad by Universidad Nacional is licensed under a CreativeCommons Reconocimiento-NoComercial- 
Los trabajos de campo, que acontecieron en diferentes momentos entre los años de 2014 a 2017 en ambos países, permitieron la recolección de información cualitativa y cuantitativa que posibilitó dar a la investigación un carácter descriptivo, comparativo, interpretativo y reflexivo, procurándose de esa forma comprender los fenómenos sociales, económicos y culturales en las realidades mexicana y brasileña.

Así pues, el trabajo de campo destaca como una metodología importantísima, pues "ofrece el diálogo entre lo teórico y lo empírico, en un proceso de constante reconstrucción" (Tedesco, 1999). Por medio de esas experiencias prácticas fue posible la aproximación con sujetos directamente ligados a los efectos de la palma africana en los espacios investigados y la comprensión de la percepción de como ellos aprehenden sus territorialidades.

Entre los instrumentos y técnicas de investigación se utilizaron: entrevistas semiestructuradas, observación participante, grabaciones de audio, conversaciones informales, registros en el diario de campo, mapas, entre otros. A continuación, pasamos a un análisis del dominio agroenergético sobre los espacios rurales, buscando brindar una contextualización más general sobre la temática $y$, posteriormente, sobre los contextos mexicano y brasileño en cuanto a la producción de la palma africana y sus efectos socioambientales.

\section{Sección primera: algunas ideas en torno de la dominación del modelo agromercantil capitalista}

\section{El dominio agroenergético de los espacios rurales}

El proceso de expansión capitalista sobre el espacio agrario se vincula también con el hecho de que la producción de alimentos para los seres humanos y animales está incorporada a un dominio agroenergético del territorio, que se relaciona con la producción de agrocombustibles, la extracción de hidrocarburos y la explotación minera. En términos reales, como afirma Gorenstein (2016), la región latinoamericana y del Caribe:

[...] exporta más de lo que importa y, en conjunto, el subcontinente representa $13 \%$ del comercio mundial de productos agrícolas, con una tasa de crecimiento de $8 \%$ anual en los últimos 20 años. El Cono Sur es un proveedor importante de la soja que sirve como insumo para la producción de carne, y se han expandido otros de los denominados "cultivos flexibles" o comodines -maíz, caña de azúcar y palma- con fines alimentarios, pero también utilizables como biocombustible. La incidencia del capital transnacional intensifica tendencias instaladas hace tiempo en ALC. Las transnacionales agroalimentarias desempeñan un papel clave en la dinámica de un proceso que incorpora diferentes territorios a las relaciones de producción y consumo global. A través de sus inversiones y modalidades organizativas, conforman complejas estructuras (redes, mallas) y al actuar en múltiples localizaciones ejercen el gobierno de diferentes eslabones de las cadenas agroalimentarias bajo marcos regulatorios y competitivos en los que se 
combinan de manera compleja instancias nacionales, regionales $y$ mundiales. (p. 1)

Las grandes corporaciones agroalimentarias, que forman parte del capitalismo global, han impactado la estructura agraria regional, impidiendo la democratización del acceso a la tierra para millones de campesinos, cooptando los aportes públicos y gubernamentales de las naciones latinoamericanas y transformando el funcionamiento de la economía agraria que está ligado al acceso a las semillas, a los agroquímicos y tóxicos, créditos, asistencia técnica, comercialización, logística y agregación de valor económico a los productos primarios.

El campo latinoamericano está bajo dominación del oligopolio genético-químico impulsada con fuerza de grandes empresas como Bayer, Dupont, Dow Syngenta, BASF y Monsanto. La revolución verde ha significado para los campesinos una nueva subordinación a estas empresas, debido a la introducción de un nuevo paquete tecnológico que contempla semillas genéticamente modificadas por las que se pagan derechos de propiedad intelectual, el uso masivo de químicos y tóxicos con la que vienen acompañados y el impulso decisivo a los productores de exportación, en detrimento de la soberanía alimentaria.

Esto último ha ocasionado una expansión de la frontera agrícola sin precedentes $y$, por consiguiente, devastación ambiental. En América Latina somos testigos de cómo cultivos como la soya, eucalipto, la caña de azúcar, la palma africana, hule, entre otros y la pecuaria capitalista causan la pérdida de grandes ecosistemas naturales, como es el caso del cerrado brasileño, de la Amazonia y del Grande Chaco en Argentina, Paraguay y Bolivia. En el caso mexicano y guatemalteco, la pérdida de la selva lacandona y del Peten es alarmante. Como afirma Gorenstein (2016):

El extendido proceso de ampliación de las tierras destinadas a cultivos agroindustriales en ALC se basa tanto en la incorporación de nuevas superficies (no previamente utilizadas para tal fin) como en el desplazamiento de otras producciones y/o segmentos de la agricultura familiar orientados a los mercados locales. Asimismo, este fenómeno ha sido impulsado por la creciente difusión de nuevos paquetes tecnológicos, la ampliación de la demanda internacional y el desarrollo de los cultivos flexibles. Este último factor, sumado a otros de carácter extrasectorial-como el desarrollo de la silvicultura forestal, la conservación a gran escala, los mecanismos de compensación de carbono y la extracción de mineraleshan tenido una incidencia significativa en la inversión extranjera destinada al acaparamiento de tierras. El crecimiento de los cultivos flexibles o "comodín" y la concentración, la extranjerización y el acaparamiento de tierras han estado muy vinculados. (p. 8)

Los procesos de concentración y de extranjerización de la tierra están presentes, principalmente, en Argentina, Brasil, Colombia, Ecuador, Paraguay, Perú, Uruguay y Guatemala. Según la información de la Comisión Económica para América Latina (CEPAL, 
$\underline{2013}$ ), los grandes inversionistas extranjeros en América Latina provienen del Golfo Pérsico, China y Corea del Sur (en Argentina y Brasil), EUA y países europeos (en Colombia, Perú y México), Japón (en Brasil, Colombia y Ecuador). Regionalmente, el capital mayoritario brasileño invierte en Mercosur y en Chile. Las empresas transnacionales de capital mayoritariamente mexicano, en América Central y en Colombia.

Siendo así, las grandes empresas agroalimentarias, en las que participa una clase capitalista transnacional, fueron las grandes beneficiarias del "boom" de las commoditties en las bolsas del mercado futuro y del apoyo gubernamental para extender sus estrategias de alianzas y fusiones. Dentro de esta estrategia de dominio territorial de concentración de tierras y de territorios se dan los apoyos fiscales, créditos, subordinación de mercados y el aumento de lucro de las empresas transnacionales, todo insertado en el proceso de crecimiento de las plantaciones de la palma africana en los dos países.

\section{Sección segunda: la investigación y sus resultados}

\section{La palma africana en México}

México tiene un gran déficit en la oferta de aceite de palma para satisfacer la demanda interna. En 2015, el país produjo 118,724 Tm de aceite de palma crudo (APC), que tan solo satisface el $20 \%$ del consumo del país, y 10,502 Tm de aceite de palma (PKO), equivalente al $16 \%$ del consumo nacional. El consumo interno total asciende a 583,466 Tm de APC y 62623 Tm de PKO, según datos de la Secretaría de Agricultura del Gobierno Federal mexicano (SIAPSAGARPA, 2017).

En respuesta a la encumbrada demanda interna y a las oportunidades de exportación, el Estado mexicano en todos sus niveles de gobierno ha promovido políticas públicas para mejorar la producción mediante el financiamiento y la modernización de los procesos de producción.

Por ejemplo, desde el año 2010 la Secretaría de Agricultura del Gobierno de México estableció subsidios a través del programa "Trópico Húmedo" -hoy Programa Regional de Desarrollo Productivo Sur-sureste-, que proporciona un 30\% de los valores de establecimiento del cultivo de Palma Africana y que es dispersado mediante el Fideicomiso Instituido en Relación con la Agricultura (FIRA) del Banco de México. A ello se suma el Fideicomiso de Riesgo Compartido (FIRCO) de la SAGARPA, que permite canalizar recursos públicos, privados o mixtos hacia a proyectos productivos relacionados con esta planta.

Para el año 2013 se incorporaron las Alianzas Productivas, mediante las cuales los grandes productores de palma africana o empresas agroindustriales pueden incorporar a los campesinos en sus proyectos de ampliación de hectáreas del cultivo, creación de viveros o manejo de cosecha, entre varias actividades. Si se inscribe un proyecto solo como empresa, se le otorga el $20 \%$ del subsidio del proyecto; si lleva una alianza con campesinos, el 50\%, y si 
el pequeño productor familiar va solo, el $70 \%$. Todo ello como subsidios gubernamentales (Fondo Perdido) para el impulso de la palma africana en México.

A ello se ha sumado la creación de una sociedad financiera por parte de los grandes agricultores y acopiadores de la palma de aceite en México, que han constituido FOMENPALMA S.A. de C.V., una sociedad financiera de objeto múltiple (SOFOM) de carácter privado, que incluye la participación del Consejo Mexicano para el Desarrollo de la Palma de Aceite (COMEXPALMA) e industriales del ramo. Su actividad principal es el apoyo a productores de palma de aceite mediante el otorgamiento de crédito -con tasas de interés por debajo del mercado- para fines productivos.

Todo este proceso de avance "agroindustrial" se ha dado mediante un proceso de "asociacióncooptación" de la estructura corporativa campesina regional, lo que ha permitido un desarrollo consensado con los campesinos locales, tal es el caso del Soconusco, Palenque y Marqués de Comillas en el estado mexicano de Chiapas. Sin embargo, para el caso de las nuevas plantaciones en Campeche, aparecen nuevos actores con mayor capital y con grandes extensiones de tierra, que rompen la práctica del "desarrollo consensado" y que se acercan a experiencias como el land grabbing, practicado sobre todo en África, Sudamérica y Asia.

La palma africana en México se ha convertido en uno de los impulsores centrales de la deforestación de la Selva Lacandona y de las zonas tropicales del sureste mexicano; por lo tanto, es una actividad promotora del cambio climático. Ello porque el modelo de negocios que promueve, basado en la agricultura de contrato, impulsa a los campesinos a desmontar la floresta para sembrar palma africana. Lo anterior es muestra del:

Proceso de mundialización (que) ha generado la incorporación de grandes actores económicos a la producción agrícola, es así que las empresas trasnacionales han visto como un nicho de oportunidad, el cultivo de la palma africana para el abastecimiento en primer término de la industria alimentaria y de cosméticos y, en un segundo término, la conversión a biodiesel de la pasta obtenida. (Ávila, $\underline{2014}$, p. 60) 


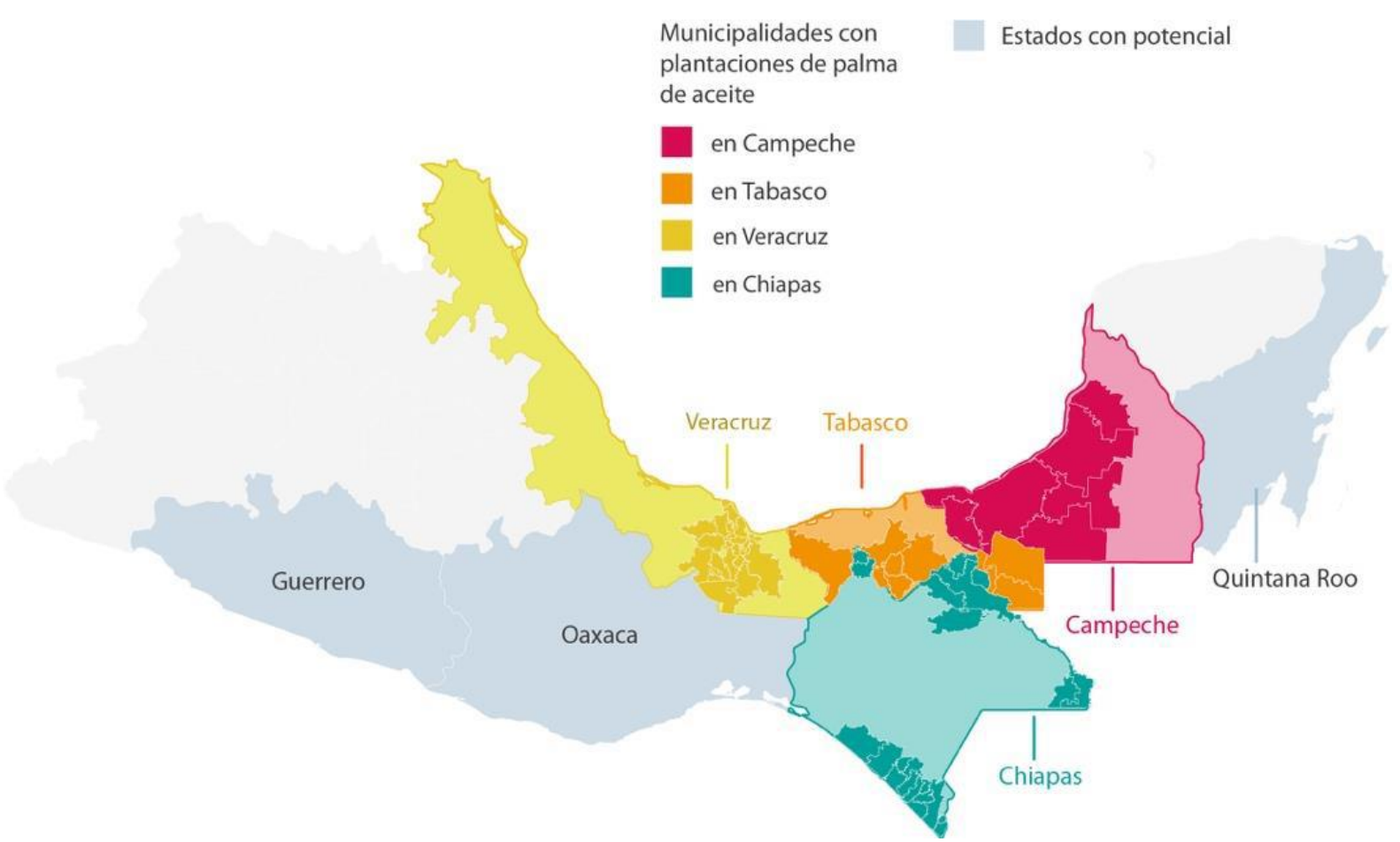

Figura 1. Municipios y estados de México con cultivo de palma africana. Fuente: Servicio de Información Agroalimentaria y Pesquera-Secretaría de Agricultura SIAP-SAGARPA (2017), Proforest.

Ello es importante visualizarlo porque, como afirma Gustavo Castro de la Organización de la Sociedad Civil Otros Mundos (2009), las empresas transnacionales son los principales responsables de los problemas que causan las plantaciones: el acaparamiento de los territorios y de los bienes comunes; la destrucción de áreas biodiversas y formas de vida asociadas; el secamiento y la contaminación por pesticidas de los ríos, arroyos y pozos; el agotamiento y la erosión del suelo; condiciones de trabajo degradantes; y un creciente proceso de financiarización ${ }^{3}$ de la naturaleza sobre las tierras y la producción. Sin embargo, estas empresas no solo persisten en negar y encubrir sistemáticamente todos estos procesos de injusticia social y ambiental, sino que también se consideran parte de las 'soluciones' a estos problemas. Algunas de las falsas soluciones de mercado o, más bien, las soluciones para el propio capitalismo financiero aumentan las injusticias asociadas a los monocultivos, con una serie de iniciativas que legitiman las operaciones empresariales, sin obligarles a rendir cuentas por los crímenes y violaciones cometidos.

En estudios anteriores (Ávila et al., 2014; 2015) se señalan tres grandes impactos que dicha actividad forestal genera: 1) la concentración del ingreso en pocas empresas y transferencias de subsidios gubernamentales a grandes agentes privados, 2) la gran utilización de fertilizantes y agroquímicos, con lo que se contribuye enormemente al cambio climático y se

\footnotetext{
${ }^{3}$ Se entiende la financiarización como el proceso de la economía mundial donde se privilegia el capital financiero trasnacional en detrimento del capital productivo y de participación del Estado, fomentando la concentración del ingreso en el complejo financiero bancario y en los sectores extractivos y energéticos (Robinson, 2011).
} 
elimina de manera extraordinaria la biodiversidad que caracteriza al estado de Chiapas y 3) una imposición colonial-cultural a la vida campesina e indígena, ya que los productores son subordinados como simples trabajadores de la agroindustria capitalista.

En relación con las relaciones de trabajo y de vida de las personas, los cultivos de palma africana en México son señalados por causar graves efectos ambientales, como la contaminación del agua por el uso de agroquímicos o la privatización de esta por las grandes cantidades que se requieren para este cultivo. Asimismo, en cuestiones laborales se denuncia comúnmente la violación de los derechos de los trabajadores de la palma, ya que tienen que desarrollar trabajos físicos extremos, con insuficiente hidratación e inadecuado suministro y uso del equipo de protección personal. Se menciona también que los trabajadores no cuentan con un salario fijo, sino que este depende fundamentalmente de alcanzar la meta de recolección, lo que implica jornadas extenuantes de trabajo por alrededor por 8 dólares diarios. Además, es común el trabajo infantil en actividades peligrosas.

A ello se suman algunos impactos que el cultivo de palma africana viene provocando sobre los espacios rurales, entre los que destacan:

1. Mudanzas en la propiedad de la tierra hacia las agroempresas (en vez de propiedad comunal o individual) y un aumento del control del paisaje productivo por las agroempresas (mediante el arrendamiento de tierras, el abastecimiento de semillas, la intervención de las plantaciones, etc.).

2. Variaciones graduales en el paisaje productivo sin tener en cuenta los efectos a gran escala de estos cambios y sin una consulta adecuada sobre los impactos (en parte porque se trata de múltiples cambios de pequeña escala en el uso de la tierra, en lugar de una única adquisición).

3. Dependencia de cultivos comerciales, a costa de los cultivos de manutención o de consumo local, lo que plantea interrogantes sobre la seguridad alimentaria local a largo plazo y la posible vulnerabilidad económica.

4. Una denuncia grave de desalojo de poblaciones de sus tierras para el establecimiento de plantaciones o plantas extractoras de aceite de palma en el estado de Campeche.

Lo importante es anotar el crecimiento espectacular que está teniendo el cultivo de la palma africana en México, pasando de 49,000 hectáreas sembradas en el año 2010 a más de 90,000 actualmente, ampliándose la frontera agrícola a través de la destrucción de la cubierta forestal tropical y el despojo a campesinos e indígenas. 
Tabla 1.

Palma africana en México. Superficie sembrada (hectáreas) por estado

\begin{tabular}{ccc}
\hline Entidad federativa & $\mathbf{2 0 1 0}$ & $\mathbf{2 0 1 6}$ \\
\hline Chiapas & $33,500.48$ & $43,443.70$ \\
Campeche & $3,715.00$ & $23,228.00$ \\
Tabasco & $5,939.91$ & $16,195.24$ \\
Veracruz & $6,426.50$ & $7,151.30$ \\
TOTAL & $\mathbf{4 9 , 6 6 1 . 8 9}$ & $\mathbf{9 0 , 1 1 8 . 2 4}$ \\
\hline
\end{tabular}

Fuente: Servicio de Información Agroalimentaria y Pesquera (2017), elaboración propia.

Se trata de un negocio creciente donde a la deforestación que se produce se le suma la alta utilización de agentes químicos y tóxicos y la superexplotación a la que son sometidos los trabajadores en la cosecha del producto.

Estás más de 90,000 hectáreas de palma africana involucran ya en México a más de 12,000 palmicultores, de los cuales la mayoría no ha encontrado los beneficios económicos que se les prometieron y muchos de ellos continúan sobreviviendo en condiciones de marginación económica y social. Por ejemplo, el estado de Chiapas produce más del $60 \%$ de la palma africana y es el estado con mayor población en la pobreza de todo México, con más del $76 \%$ de sus habitantes.

A ello se suma la concentración de tierras con este monocultivo, como los que se han desarrollado en Tabasco con la Agroforestal Uumbal, la cual ha plantado 1,487 Ha, la empresa Proteak con 1,300 Ha, Palmosur con $500 \mathrm{Ha}$ y Propalma con $600 \mathrm{Ha}$, en el estado Chiapas. Según datos COMEXPALMA (2016) el 70\% del suministro del fruto viene de productores individuales organizados y el otro $30 \%$ proviene de plantaciones propias de la agroindustria. En México existen 11 empresas extractoras de palma de aceite que funcionan al $50 \%$ de su capacidad instalada.

Tabla 2.

Empresas extractoras de aceite de palma en México

\begin{tabular}{ll}
\multicolumn{1}{c}{ Estado } & \multicolumn{1}{c}{ Empresa } \\
\hline Chiapas & El Desengaño, Bepasa, Propalma, Zitihualt, Palmicultores, Palmatica \\
Veracruz & Aceites de Palma \\
Campeche & Oleofinos del Carmen \\
Tabasco & Agroimsa, Agroipsa \\
\hline
\end{tabular}

Fuente: elaboración propia con datos de COMEXPALMA (2016).

Es de relevancia anotar que alrededor del lugar geográfico que abarcará la Zona Económica Especial de Puerto Chiapas y la Zona Económica Especial de Coatzacoalcos es donde se han impulsado los cultivos de este bien y la construcción de plantas agroindustriales para su posterior transformación (Ávila y Ávila, 2016 y 2017). Ello es relevante porque alrededor de 
América Latina encontramos diferentes ejemplos de instalación de complejos agroindustriales dedicados a la producción de aceite de palma africana que hacen uso de las facilidades logísticas, fiscales, aduaneras y gubernamentales que les proporcionan las ZEE, como nos muestran los casos de Honduras, Colombia y Ecuador. Las ZEE se constituyen así en un instrumento dentro de la cadena de valor palmífera para facilitar los procesos de circulación del aceite de palma y, con ello, aumentar la ganancia de los empresarios involucrados.

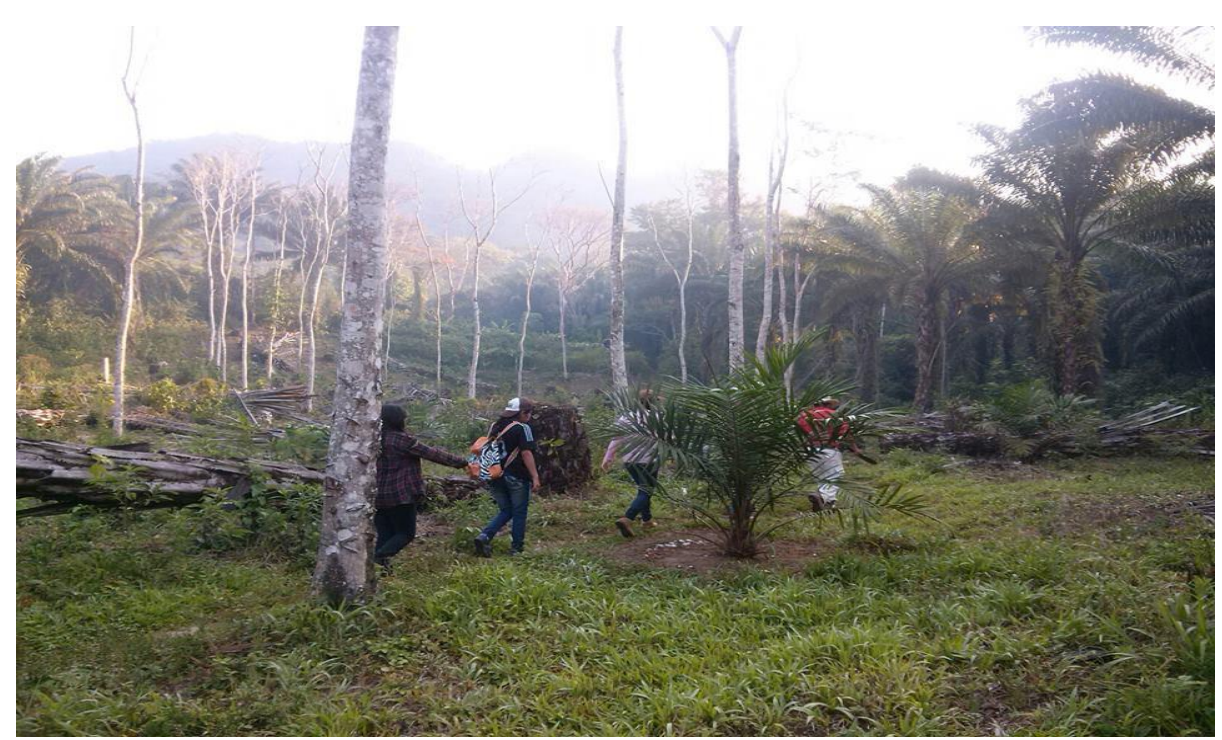

Figura 2. Deforestación por cultivo de Palma Africana. Comunidad Vicente Guerrero, Chiapas, México. Fuente: Agustín Ávila. Trabajo de Campo.

\section{La Palma africana en Brasil}

Las primeras semillas de la palma africana fueron introducidas en Brasil por barcos de esclavos procedentes del continente africano y se desarrollaron en el litoral sur de la Bahía a mediados del siglo XVI. Conocida comúnmente en Brasil como donde, el aceite de palma es famoso por ser uno de los principales ingredientes de la cocina baiana y de la cultura afrodescendente (Watkins, 2011); mientras tanto, en la Amazonia, la producción de la palma africana, básicamente toma dos destinos específicos: para la industria alimentaria y farmacéutica y, más recientemente, para la producción del agrocombustible. 


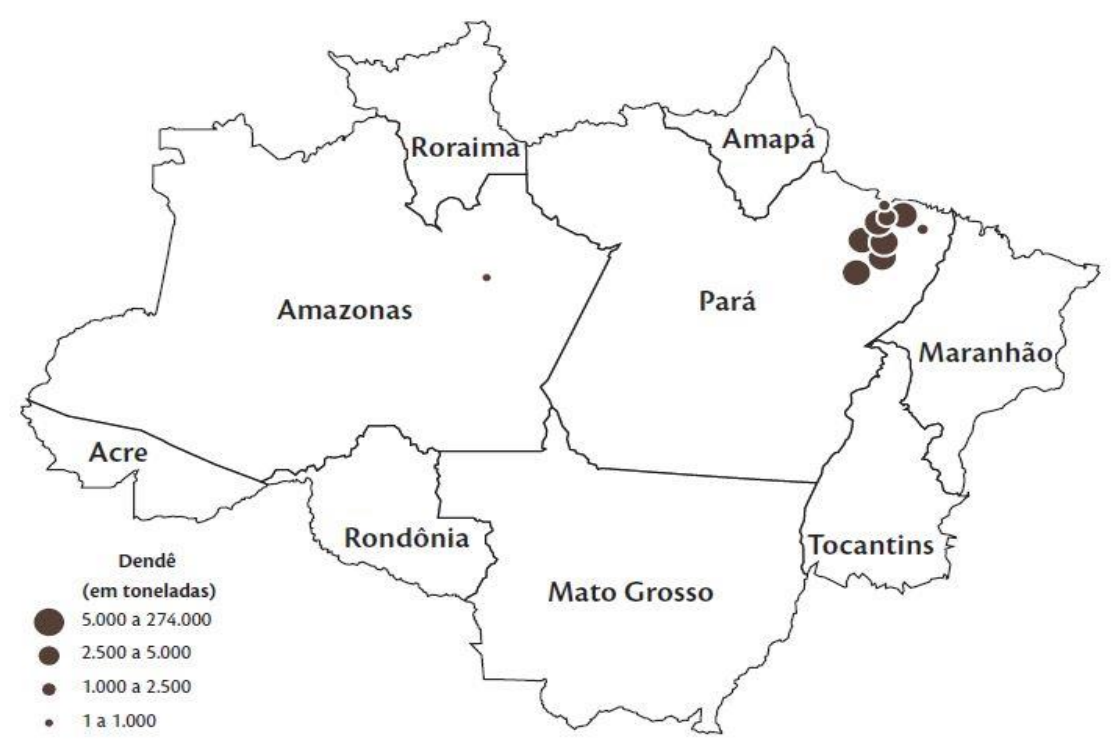

Figura 3. Producción de aceite de palma, Brasil, 2006. Fuente: Centro de Gestión y Estudios Estratégicos, $\underline{2009}$.

La producción de palma en Brasil se centra en la Amazonia, específicamente, en el estado de Pará, responsable por casi $90 \%$ de la producción nacional. En 2017 , la producción brasileña de aceite de palma llega a 410,000 toneladas (Departamento de Agricultura de Estados Unidos, 2017). En Pará, la producción se centra en nordeste del estado, formando el llamado "polo de producción del dendê", construido por la actuación de las siguientes empresas y municipios, conforme a la Tabla $\underline{3}$.

Tabla 3.

Empresas y municipios productores de dendê en Pará

\begin{tabular}{ll}
\hline Empresas & Municipios \\
\hline Agropalma & Acará, Moju e Tailândia \\
Biopalma & $\begin{array}{l}\text { Abaetetuba, Acará, Concórdia do Pará, Moju, Tomé-Açu e São Domingos } \\
\text { do Capim }\end{array}$ \\
Yossan & Santa Izabel do Pará \\
Denpasa & Santa Bárbara do Pará \\
Marborges & Moju \\
Dentauá & Concórdia do Pará, Santo Antônio do Tauá \\
BBB/Petrobrás/Galp & Moju, Tailândia, Tomé-Açu, Acará, Concórdia do Pará, Bujaru e \\
ADM & Abaetetuba \\
Palmasa & São Domingos do Capim \\
\hline
\end{tabular}

Fuente: elaboración propia con datos de Repórter Brasil (2013). 
La palma llega a la Amazonia en la década 1950, por iniciativa del Instituto Agronómico del Norte (IAN), predecesor de la Empresa Brasileña de Investigación Agropecuaria (EMBRAPA), con el objetivo, en principio, de probar su viabilidad en el suelo y clima amazónicos. En la década siguiente, la Superintendencia del Desarrollo de la Amazonia (SUDAM), en asociación con el Institut de Recherches pour les Huiles et Les Oleagineux (IRHO), establecen cultivos para evaluar su viabilidad económica. En la década de 1970 marca el paso de las plantaciones para la iniciativa privada, seguida de la dinamización de la producción de palma en Pará. Hasta la primera década de los años 2000 la producción de aceite de palma se destinaba casi exclusivamente a las industrias de alimentos y farmacéutica. Actualmente, la producción todavía es predominantemente destinada para estos dos sectores, añadiéndose el sector de energía con los agrocombustibles.

La crisis del petróleo de la década de 1970 da inicio a la búsqueda de fuentes alternativas de energía, que también se extiende a un debate mundial sobre la cuestión ambiental. Brasil, ya en la década de 1970, con el Programa Nacional del Alcohol, asume una postura que señaló el cambio de su matriz energética. En 2004, con la creación del Programa Nacional de Producción y el Uso del Biodiesel (PNPB) vuelve a la superficie el debate acerca de los agrocombustibles que, esta vez, trae una especificidad, la "inclusión social" de los campesinos en la cadena productiva, siendo una directriz relevante en el ámbito de este programa. Mientras tanto, nuestra interpretación es que el PNPB ha reproducido la fórmula consagrada de política pública brasileña de brinda formas de acceso a la renta por el sesgo de la reproducción del capital, sin modificaciones estructurales (Fernandes, 2008). Esta es una cuestión fundamental, porque la cuestión agraria en Brasil nunca se ha resuelto; el país presenta una alta concentración agraria y tierras, en su mayoría, arrebatadas o grilladas.

Backhouse (2013), realizando un análisis sobre los agrocombustibles en contexto mundial, afirma que en la Amazonia la producción del aceite de palma está centrada en "triple ganancia"; esto es, dar cuenta de la protección ambiental, desarrollo rural y del propio sector de aceite de palma. La autora concluye que, por medio de la actuación del Estado, contrariando el discurso de sustentabilidad, la producción de aceite de palma ejerce serios impactos ambientales, además de apropiarse localmente de las tierras, dando cuerpo al fenómeno "green grabbing".

En el acto mismo de sus consecuencias este proceso es legítimo ideológicamente por la doctrina neoliberal, y gana así contornos de proyecto hegemónico. Por esa razón, concebimos el green grabbing como un proceso también discursivo. En el adjetivo "green" (verde) se anhela un creciente consenso de naturaleza neoliberal que traspasa transversalmente el campo político y preconiza la mercadería de recursos naturales como el medio más eficaz de protegerlos. (Corson y Macdonald, 2012, citado en Backhouse, 2013, p.10)

En 2010 es lanzado el Programa Nacional de Producción Sustentable de Aceite de Palma (PPSOP) que, reforzando el PNPB, tiene como objetivo ordenar la producción de aceite de 
palma en Brasil, estableciendo líneas de crédito para la producción y limitando en áreas antropizadas, esto prohíbe la deforestación para la creación de nuevas áreas de cultivo.

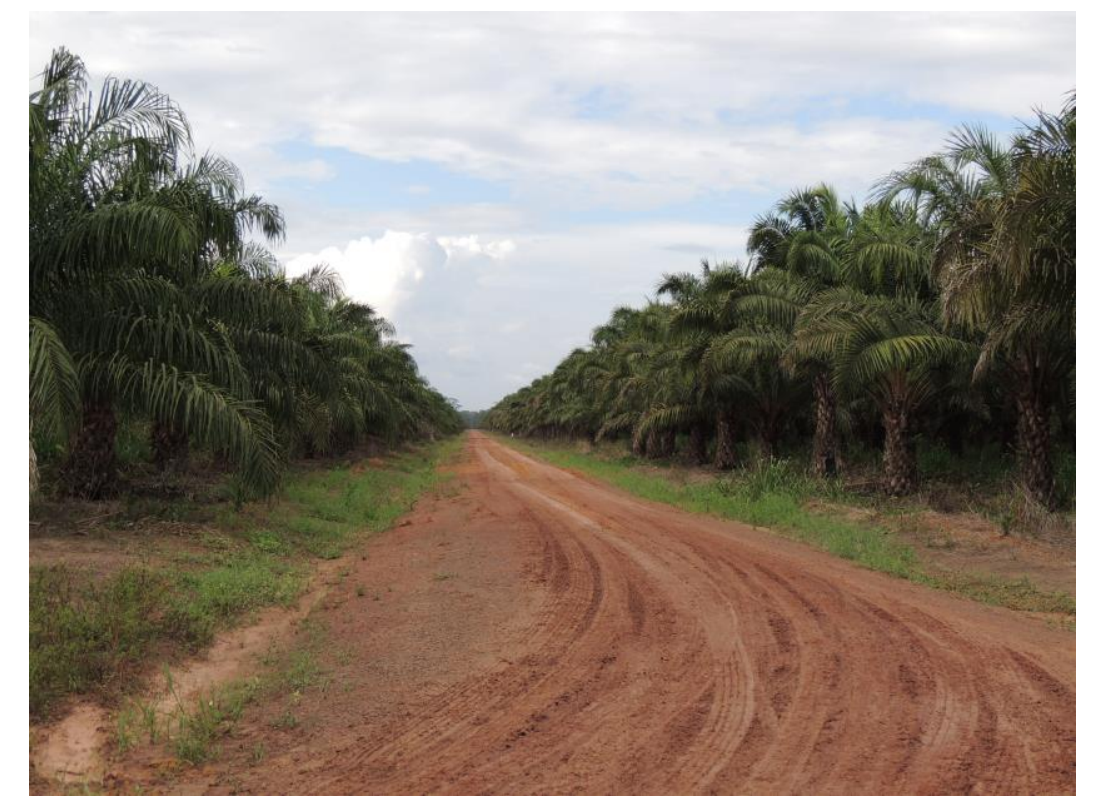

Figura 4. Las Palmas de aceite en Tomé-Açu, Pará, Brasil, 2017. Fuente: trabajo de campo.

En ese sentido, teniendo en vista las especulaciones que se crearon entorno de ese proyecto de producción de palma en el nordeste de Pará, lo que se asistió localmente fue una competencia por la compra de tierras, alterando su precio, y una especie de fraude para que los campesinos vendieran sus tierras a los empresarios, afectando, inclusive, territorios cimarrones, como aconteció en 2008 en Concordia del Pará, con la participación de la empresa Biopalma (Repórter Brasil, 2010). Según la Asociación Brasileña de Productores de Aceite de Palma (ABRAPALMA), en 2014, el área plantada en la mesoregión nordeste paraense era de 178,073 hectáreas, de los cuales 30,461 estaban en 1,124 unidades campesinas por medio de la integración a la agroindustria de la palma (ABRAPALMA, 2014).

Por tanto, estamos frente a dos procesos que operan simultáneamente, relacionados con la producción del aceite de palma en Pará: la territorialización del monopolio y la monopolización del territorio por el capital (Oliveira, 2010). El primero se da por medio de la compra de tierras y el segundo, por la integración de los campesinos a la agroindustria de la palma.

Lo que viene ocurriendo actualmente, por lo tanto, en el ámbito de la integración, una manera que el capital monopolista encontró de desarrollarse en esta parte de la Amazonia; y para eso no ha prescindido solamente de relaciones típicamente capitalistas, sino que está ante un proceso de subordinación de la producción campesina por medio de la apropiación de la renta de la tierra (Oliveira, 2010, citado en Albuquerque y Oliveira, 2017, p. 161). 
La monopolización se describe como un proceso continuo de pérdida de la autonomía campesina, en la cual estos sujetos van perdiendo la libertad de plantar lo que quisieran en su propiedad porque ahora necesitan seguir las directrices repasadas por la agroindustria; es decir, interfiriendo en el proceso y en el ritmo de su trabajo y de su familia, alterando la lógica interna de la unidad campesina. Asimismo, la producción de la palma en la unidad campesina ha significado una alteración en el sistema de producción familiar, introducción de maquinarias y agrotóxicos, disminución en las áreas de rozas y producción de la yuca (Silva \& Navegantes-Alves, 2017b); se presenta un cuadro de desproporcionalidad de incentivos públicos en relación con la producción de la palma y la producción de cultivos de alimentos, como la yuca, se tiene como resultado que la primera tiene mayor adhesión de los campesinado $\mathrm{y}$, consecuentemente se presenta una disminución de la producción de alimentos (Mota et al., 2015).

Por último, al ser la palma africana una estrategia del Estado, las empresas logran un dominio territorial agroindustrial formando un "latifundio verde", una concentración agraria (Silva \& Navegantes-Alves, 2017, p. 17); además de ser esto, en realidad, una violación de la legislación vigente por medio de la incorporación de algunas áreas al agronegocio de la palma (Silva, Magalhães, \& Farias, 2016).

Por su parte, los impactos ambientales son notorios en cuanto al cultivo de la palma. Según el reporte de Repórter Brasil (2010), la palmera necesita de muchos agroquímicos para producir una cantidad rentable de aceite $y$, en la Amazonia, con su clima lluvioso, esto tiene consecuencias graves, porque estas sustancias son fácilmente llevadas al curso de agua, pudiendo causar alteración química de los ríos y un impacto inmediato a los peces y vegetación acuática.

En suma, la producción de palma en Brasil y, especialmente en la Amazonia, teniendo la presencia marcante del Estado, ha significado una reedición de los proyectos hegemónicos que la zona ya ha presenciado desde la década de 1970; son concepciones economicistas articuladas y pensadas de fuera de la región en la que figura como recurso natural de agregación y dominación de arriba hacia abajo, por la lógica capitalista.

\section{Reflexiones finales}

El cultivo de palma africana en México y Brasil ha sido fuertemente impulsado por parte de políticas públicas gubernamentales, que trasladan recursos dirigidos a los pequeños productores -fundamentalmente campesinos e indígenas- para la conformación de un sistema de plantaciones ligadas a la lógica agroindustrial y de funcionamiento del capitalismo contemporáneo. Eso es lo que se ha denominado la territorialización de los monocultivos y de la dinámica monopólica del capital.

Como hemos anotado, ello ha dado origen a la conformación de nuevos grupos empresariales ligados fundamentalmente al acopio, extracción y transformación de la palma africana; 
además, ha traído consecuencias devastadoras sobre la biodiversidad y las dinámicas campesinas e indígenas de los dos países.

A nivel ambiental se mencionan graves efectos del cultivo que tienen que ver con la utilización de un paquete tecnológico que utiliza una gran cantidad de químicos y provoca gases de efecto invernadero que contribuyen al calentamiento global. A ello se suma la imposibilidad de las aves para poder anidar y a que muchas de estas plantaciones se ubican en espacios de tránsito de diferentes especies animales, como los monos o el jaguar, por ejemplo. Además, la utilización de grandes cantidades agua que precisa este monocultivo es algo denunciado por ambientalistas también.

Asimismo, se menciona, a nivel social, los bajos salarios que reciben los trabajadores de la palma y cómo los ingresos económicos de los palmicultores, en el caso mexicano, no les ha permitido superar las condiciones de marginación y pobreza en la que viven. A ello se suman estrategias empresariales para el despojo agrario de las tierras de campesinos e indígenas para el impulso de este cultivo.

Como se demostró, la expansión y la actuación del sector de palma en los dos países han significado una alteración de las formas de organización de la agricultura campesina, violación de derechos y de la legislación y el establecimiento del propio capitalismo en el campo, frente al agronegocio. En ambos países podemos observar grandes similitudes en cuanto a la actuación del sector. Se resalta la serie de riesgos impuestos por esta actividad, así como un amplio proceso de territorialización de los monopolios y de monopolización del territorio, este último, en el caso brasileño, por la integración de los campesinos a la agroindustria del dendê.

Podemos afirmar que estamos ante los holdings, o sea, fusión de capital inscrita en su reestructuración productiva, garantizando siempre su reproducción ampliada. En América Latina se ha asistido a un intenso proceso de explotación del campo y expropiación de campesinos e indígenas, casi siempre atado a proyectos hegemónicos que se basan en estrategias como el discurso del desarrollo y la sostenibilidad; es decir, llevando a cabo un discurso ideológico, como es el caso del green grabbing, al mismo tiempo que tiene en su modus operandi el aumento de la concentración de tierra y el incumplimiento de los acuerdos (land grabbing).

Finalmente, se hace necesario la profundización de los análisis sobre la actuación del sector de la palma no solo en Brasil y México, sino en todo el mundo, sobre todo en la región latinoamericana, donde se apunta el crecimiento de la producción y del área plantada de palma y el establecimiento de los grandes monopolios agroenergéticos. Además de buscar comprender el papel del campesinado, sus formas de cooptación y reproducción ante la actuación de la agricultura capitalista y el capital mundializado. 


\section{Referencias}

Asociación Brasileña de Productores de Aceite de Palma. (2014). Planilha de mapeamento da palma de óleo no Brasil. Pará, Associação Brasileira de Produtores de Óleo de Palma.

Albuquerque, J. y Oliveira, A. (2017). Os efeitos socioeconômicos da parceria entre a agroindústria do dendê e a agricultura camponesa em Tomé-Açú, Pará. Recuperado

http://www.enanpege.ggf.br/2017/anais/arquivos/GT\%2038/1368.pdf

Ávila, A. (2014). La Reserva de la Biosfera de la Sepultura y el deterioro biocultural por la privatización de la naturaleza. In. ALBERTO BETANCOURT, et. al. (Coord.). Libro: Del Monologo a la Polifonía. Proyectos supranacionales y saberes indígenas en la gestión de áreas naturales protegidas (1990-2010). UNAM-FFyL CONACYT. México. Recuperado de https://territoriosypatrimonio.files.wordpress.com/2018/02/a betancout etal del monologo polifonia 2015-1.pdf

Ávila, A. y Ávila, L. E. (2016). Reconfiguraciones territoriales y neoextractivismo: la nueva Zona Económica Especial de Chiapas, México. Revista Cardinalis de la Universidad de Córdoba, (7), 4-34. Recuperado de https://revistas.unc.edu.ar/index.php/cardi/article/view/16249

Ávila, A. y Ávila, L.E. (2017). Las Nuevas Zonas Económicas Especiales en México: despojo agrario y resistencia campesina. Revista Núcleo de Estudos, Pesquisas e Projetos de Reforma Agrária de la Universidade Estadual Paulista, 40(20), 138-162. Recuperado de http://revista.fct.unesp.br/index.php/nera/issue/view/n.\%2040

Ávila, L. (2011). Chiapas entre la protección ambiental y la producción alimentaria: el impacto de los agro combustibles. En L. Ávila. Desarrollo Sustentable, Interculturalidad y vinculación comunitaria. (1 ed, 169-186). Guadalajara, Jalisco: Editorial UNICH. Recuperado de: http://bibliotecasibe.ecosur.mx/sibe/book/000050304

Ávila, L. E. y Ávila, A. (2015). Los agrocombustibles y el crecimiento verde en Chiapas, Mexico. Revista GeoNordeste de Universidade Federal de Sergipe, (1), 249-273. Recuperado de https://seer.ufs.br/index.php/geonordeste/article/view/4467/pdf

Backhouse, M. (2013). A desapropriação sustentável da Amazônia: O caso dos investimentos em dendê no Pará. (Fair Fuels? Working Paper 6). Recuperado de http://www.fairfuels.de/data/user/Download/Ver\%C3\%B6ffentlichungen/FairFuelsWorking Paper 6 Portuguese.pdf 
Castro, G. (2009). La palma africana en México. Los monocultivos desastrosos. Otros Mundos. Recuperado de http://otrosmundoschiapas.org/index.php/component/content/article/49-25-elescaramujo/49-el-escaramujo/665-la-palma-africana-en-mexico

Centro de Gestión y Estudios Estratégicos. (2009). Um projeto para a Amazônia no século 21: desafios e contribuições. Brasília, DF: Centro de Gestão e Estudos Estratégicos. Ministério da Ciência, Tecnologia e Inovações. Recuperado de https://www.cgee.org.br/documents/10182/734063/12Publica\%C3\%A7\%C3\%A3 o_Amazonia final3 COMPLETO2 6415.pdf

Comisión Económica para América Latina (2013). Estudio Económico de América Latina y el Caribe: tres décadas de crecimiento económico desigual e inestable. Recuperado de

http://repositorio.cepal.org/bitstream/handle/11362/1085/S2013574_es.pdf;jse ssionid $=62$ E0E518A9E0BBA58537229AD66932B1 ? sequence $=1$

Consejo Mexicano para el Desarrollo de la Palma de Aceite (COMEXPALMA). (2016). Importancia del cultivo de palma de aceite en México. Recuperado de https://www.comexpalma.org/2017/index.php/situacion-de-la-palma/situacionnacional-2016

Corson, C. y Macdonald, K. (2012). Enclosing the global commons: the convention on biological diversity and green grabbing. Journal of Peasant Studies, 39(2). 263-283. Recuperado https://www.tandfonline.com/doi/abs/10.1080/03066150.2012.664138

Fernandes, B. M. (2008). Conflitualidade e desenvolvimento territorial: questão agrária. En: Buainain, A. M. (org.). Libro Luta pela terra, reforma agrária e gestão de conflitos no Brasil. Campinas: Unicamp, 173-230.

Gorenstein, S. (2016). Empresas transnacionales en la agricultura y la producción de alimentos en América Latina y el Caribe. Recuperado de http://nuso.org/media/documents/Analisis_Gorenstein.pdf

Instituto Brasileño de Geografia y Estadística. (2010). População residente, por situação do domicilio e sexo, segundo as Grandes Regiões e as Unidades da Federação. Recuperado de https://censo2010.ibge.gov.br/sinopse/index.php?dados=12

Instituto Nacional de Geografia y Estadística. (2018). Encuesta Intercensal 2015. Marco conceptual.

http://www.beta.inegi.org.mx/app/biblioteca/ficha.html?upc=702825098742 
Mota, D. M., Silva, E. M., Schmitz, H., Navegantes-Alves, L. y Ferreira, M. S. G. (2015). Produção de culturas alimentares e dendê nos estabelecimentos familiares no nordeste paraense. Belém, Brasil: Universidade Federal do Pará. Recuperado de https://www.embrapa.br/amazonia-oriental/busca-de-publicacoes/-

/publicacao/1035446/producao-de-culturas-alimentares-e-dende-nosestabelecimentos-familiares-no-nordeste-paraense

Oliveira, A. U. (2010). Agricultura e Indústria no Brasil. Revista Campo-Território: Revista de Geografia Agrária, 10(5), 5-64. Recuperado de http://www.seer.ufu.br/index.php/campoterritorio/article/view/12048/7006

Programa de las Naciones Unidas para el Desarrollo. (2007). Relatório de Desenvolvimento Humano 2007/2008. Capítulo: 3. [Versión digital PDF]. Estados Unidos, New York: PNUD. Recuperado de http://hdr.undp.org/sites/default/files/hdr2007-8portuguese.pdf

Repórter Brasil. (2010). A agricultura familiar e o programa nacional de biodiesel: retrato do presente, perspectivas de futuro. Recuperado de http://reporterbrasil.org.br/documentos/AgriculturaFamiliar Biodiesel2010.pdf

Repórter Brasil. (2013). A expansão do dendezeiro na Amazônia Brasileira: elementos para uma análise dos impactos sobre a agricultura familiar no nordeste do Pará. Recuperado de http://reporterbrasil.org.br/documentos/Dende2013.pdf

Rey, C. (2013). Análisis espacial de la correlación entre cultivo de palma de aceite y desplazamiento forzado en Colombia. Cuadernos de Economía, 32(spe61), 683$718 . \quad$ Recuperado

de https://revistas.unal.edu.co/index.php/ceconomia/article/view/42494

Robinson, W. (2015). América Latina y el capitalismo global: una perspectiva crítica de la globalización. México: Ed. Siglo XXI.

Roundtable On Sustainable Palm Oil. (2017). About us. Recuperado de http://www.rspo.org/about

Servicio de Información Agroalimentaria y Pesquera (SIAP), Secretaria de Agricultura y Pesca Gobierno Federal de México SIAP-SAGARPA. (2017). Base de datos. Recuperado de http://nube.siap.gob.mx/cierre agricola

Silva, E. y Navegantes-Alves, L. (2017). A ocupação do espaço pela dendeicultura e seus efeitos na produção agrícola familiar na Amazônia Oriental. Revista Franco-Brasileira de Géographe, Confins, 30, 1-22. doi: https://doi.org/10.4000/confins.11843 
Silva, E. y Navegantes-Alves. (2017b). Transformação nos sistemas de produção familiares diante a implantação do cultivo de dendê na Amazônia Oriental. Revista Desenvolvimento e Meio Ambiente, (40), 345-364. Recuperado de http://revistas.ufpr.br/made/article/view/47330/32114

Silva, E. P., Magalhães, S. B. y Farias, A. L. A. (2016). Monocultivos de dendezeiro, capital transnacional e concentração de terras na Amazônia paraense, La monoculture d'huile de palme, le capital transnational et la concentration des terres sur l'amazonie au Pará. Revista Campo-Território: Revista de Geografia Agrária, 23(11), 165-195. doi: https://doi.org/10.14393/RCT112306

Tedesco, J. C. (1999). Terra, trabalho e familia: racionalidade, produtividade e ethos camponês. Passo Fundo: Edeupf.

Departamento de Agricultura de Estados Unidos. (2017). Oilseeds: World Markets and Trade. Recuperado de https://apps.fas.usda.gov/psdonline/circulars/oilseeds.pdf

Watkins, C. (2011). Dendezeiro: African Oil Palm Agroecologies in Bahia, Brazil, and Implications for Development. Journal of Latin American Geography, 1(10), 9-33. doi: https://doi.org/10.1353/lag.2011.0005 